Universidade de São Paulo

Faculdade de Economia, Administração e Contabilidade

Departamento de Administração

Programa de Pós-Graduação em Administração

"Estrutura Organizacional para a Realização de

Negócios Eletrônicos em Empresas Tradicionais:

Um Estudo de Caso"

Adalton Masalu Ozaki

Orientador: Prof. Dr. Eduardo P. G. de Vasconcellos

São Paulo

Outubro de 2003 
Reitor da Universidade de São Paulo

Prof. Dr. Adolpho José Melfi

Diretora da Faculdade de Economia, Administração e Contabilidade

Prof. Dra. Maria Tereza Leme Fleury

Chefe do Departamento de Administração

Prof. Dr. Eduardo P. G. de Vasconcellos 
Universidade de São Paulo

Faculdade de Economia, Administração e Contabilidade

Departamento de Administração

Programa de Pós-Graduação em Administração

\section{"Estrutura Organizacional para a Realização de Negócios Eletrônicos em Empresas Tradicionais: Um Estudo de Caso"}

Adalton Masalu Ozaki

Dissertação apresentada à

Faculdade de Economia e

Administração da Universidade de São Paulo, para concorrer ao Título de Mestre, pelo curso de PósGraduação em Administração.

Orientador: Prof. Dr. Eduardo P. G. de Vasconcellos

São Paulo

Outubro de 2003 


\section{FICHA CATALOGRÁFICA}

Elaborada pela Seção de Publicações e Divulgação do SBD/FEA/USP

Ozaki, Adalton Masalu

Estrutura organizacional para realização de negócios eletrônicos em empresas tradicionais: um estudo de caso / Adalton Masalu Ozaki. -São Paulo : FEA/USP, 2003.

$138 \mathrm{f}$.

Dissertação (Mestrado) - Universidade de São Paulo, 2003

Bibliografia.

1. Comércio eletrônico 2. Estrutura organizacional 3. Descentralização administrativa 4. Internet I. Faculdade de Economia, Administração e Contabillidade da USP II. Título.

$$
\text { CDD - 658.05678 }
$$




\section{Dedicatória}

Aos meus pais, a minha mais profunda gratidão pela educação e pelos valores que me foram transmitidos. Se consegui galgar mais esta etapa em minha vida, foi graças a vocês. 


\section{Agradecimentos}

Gostaria de prestar o meu mais sincero agradecimento às seguintes pessoas:

Prof. Dr. Eduardo Vasconcellos, pela oportunidade de trabalharmos juntos, e pelos ensinamentos que foram muito além da orientação deste trabalho.

> Adriana Obara, por todo o apoio dado durante o mestrado e por compreender minha ausência em momentos difíceis de sua vida.

Cassiano Tanabe e Silvio Hirasike, meus sócios na ADF Consulting, por terem tocado a empresa durante minha ausência nesta longa jornada que foi $o$ mestrado (espero que esta ausência não motive nenhum dos dois a deixar a sociedade ...).

> A todas as pessoas da empresa pesquisada, em especial ao Gerente de eCommerce e ao Gerente de Tecnologias Web, que abriram todos contatos e, ao final, ainda revisaram o texto, contribuindo para aumentar a confiabilidade da pesquisa. Iniciativas como esta, de apoio a trabalhos acadêmicos, certamente só têm a contribuir para que a ciência da administração avance no Brasil.

$>$ A todos os demais professores da FEA-USP que têm contribuído para minha formação desde a graduação.

Sem a colaboração, compreensão e apoio de vocês, este trabalho não seria possível. 


\section{Sumário}

Lista de Figuras ___ iv

Lista de Quadros __ v

$\underline{\text { Resumo }}$

Abstract vii

1. $\quad$ Introduçã

1.1. Caracterização da Situação Problema

1.2. Definicão do Problema __ 6

1.3. Objetivos da Pesquisa

1.4. Importância do Estudo __ 11

2. $\quad$ Revisão da Literatura 13

2.1. Estrutura Organizacional —u 13

2.1.1. Evolução da Teoria sobre Estrutura Organizacional __ 13

2.1.2. Novas Alternativas de Estrutura Organizacional __ 20

2.1.3. Estrutura Organizacional e Vantagem Competitiva ___ 22

2.1.4. A Questão da Descentralização 25

2.2. Negócios Eletrônicos ___ 32

2.2.1. Internet: Histórico e Conceitos __ 32

2.2.2. Ambiente de Negócios Eletrônicos _ 34

2.2.3. Negócios Eletrônicos e Vantagem Competitiva ___ 41

2.3. Estrutura Organizacional para Negócios Eletrônicos ___ 45

2.3.1. Importância da Estrutura Organizacional para NE __ 45

2.3.2. $\quad$ O Modelo de Moore __ 48

2.3.3. O Modelo de Gulati \& Garino

2.3.4. O Modelo de Vasconcellos \& Muritiba __ 53

2.3.5. Considerações Sobre os Modelos Pesquisados___ 54

3. Metodologia de Pesquisa

3.1. Desenho do Estudo

3.2. Escolha do Método de Estudo de Caso __ 
3.3. Unidade de Análise e Amostragem

3.4. Protocolo do Estudo de Caso 62

3.4.1. Procedimentos de Campo __ 63

3.4.2. Questões do Estudo de Caso ___ 64

3.4.3. Guia para o Relatório do Estudo de Caso ___ 65

3.5. $\quad \underline{\text { Representatividade, Validade e Confiabilidade da Pesquisa }} \_66$

4. Apresentação do Estudo de Caso 68

4.1. Apresentacão da Empresa __ 68

4.2. Os Negócios Eletrônicos na Empresa X

4.2.1. Iniciativas de Negócios Eletrônicos __ 71

4.2.2. O Processo de Adoção dos Negócios Eletrônicos __ 77

4.3. Estrutura Organizacional para NE

4.4. Análise Sobre os Graus de Descentralização de NE

4.4.1. Identificação dos Graus de Descentralização e dos Critérios para Análise

para o Caso da Empresa $X$ 는 85

4.4.2. Consolidação dos Dados Coletados _ـ 90

4.4.3. Análise da Alternativa 1: CE centralizado __ 95

4.4.4. Análise da Alternativa 2: CE incorporado em TI _ 100

4.4.5. Análise da Alternativa 3: CE descetralizado pelas BUs ____ 104

5. Considerações Finais 108

5.1. Introducãa

5.2. Conclusões

5.2.1. Iniciativas de NE Adotadas ___ 111

5.2.2. Evolução da Estrutura Organizacional para NE ___ 114

5.2.3. Análise Dos Graus de Descentralização de NE ___ 118

5.3. Limitações e Sugestões para Estudos Futuros ___ 121

5.3.1. Limitações da Pesquisa___ 121

5.3.2. Sugestão para Estudos Futuros ___ 123 
Anexo A-Roteiros de Entrevista 130

Roteiro de Entrevista da Fase I 130

Q1 - Levantamento de Informações Gerais 130

Roteiros de Entrevista da Fase II 131

Q2 - Identificacão dos Critérios para Análise da Centralização x Descentralização 131

Roteiros de Entrevista da Fase III 133

Q3 - Análise dos Graus de Descentralização 133

Material de Apoio ao Roteiro "Q3 - Análise dos Graus de Descentralização" 134

Q4 - Roteiro para Levantamento das Atividades e Decisões 136

$\underline{\text { Anexo B - Literatura sobre Internet e negócios }}$ 137 


\section{Lista de Figuras}

Figura 1.1: Centralização x Descentralização da Unidade/Departamento de NE __ 8

Figura 1.2: Centralização x Descentralização de Atividades e Decisões ___ 8

Figura 2.1: Condicionantes da estrutura organizacional segundo Vasconcellos __ 15

Figura 2.2: Desenho organizacional segundo Galbraith___ 16

Figura 2.3: As cinco partes das organizações segundo Mintzberg ___ 18

Figura 2.4: Estrutura organizacional segundo Vasconcellos e Hemsley___ 19

Figura 2.5: A Cadeia de Valores Genérica de Porter __ 22

Figura 2.6: Continuum de Centralização x Descentralização ___ 26

Figura 2.7: Ambiente de Negócios Eletrônicos __ 34

Figura 2.8: Negócios na Era Digital __ 36

Figura 2.9: Modelo de Constituição e Funcionamento de Mercados Eletrônicos___ 38

Figura 2.10: Nivel de Utilização de CE no Mercado Brasileiro __ 39

Figura 2.11: Percentual do Faturamento Gerado por Negócios Eletrônicos ___ 39

Figura 2.12: Aplicações Proeminentes da Internet na Cadeia de Valor ___ 43

Figura 2.13: Evolução nos Modelos de Negócios Eletrônicos segundo Moore __ 50

Figura 2.14: Spectrum de “Cliques e Cimento” segundo Gulati \& Garino ___ 51

Figura 3.1: Metodologia para Estudo da Estrutura Organizacional de NE __ 58

Figura 4.1: Organograma da Empresa $X$

Figura 4.2: Solução de VMI na Empresa $X$

Figura 4.3: EDI utilizado na Empresa X_ 75

Figura 4.4: Beneficios Potenciais de Tecnologias para Transação entre Empresas_ 77

Figura 4.5: Organograma da Gerência Responsável pelas Aplicações de NE___ 83

Figura 4.6: CE Centralizado (A1) __ 85

Figura 4.7: CE Incorporado em TI (A2) ___ 86

Figura 4.8: CE Descentralizado (A3)___ 86

Figura 4.9: Critérios pelos quais a alternativa A1 foi melhor avaliada ___ 98

Figura 4.10: Critérios pelos quais a alternativa A2 foi melhor avaliada __ 101

Figura 4.11: Critérios pelos quais a alternativa A3 foi melhor avaliada ___ 106

Figura 5.1: Distribuição das Atribuições de NE pela Empresa ("Oficial”) ___ 114

Figura 5.2: Distribuição das Atribuições de NE pela Empresa (Praticada) ___ 115 


\section{Lista de Quadros}

Quadro 2.1: Fatores Para Análise de Centralização x Descentralização 28

Quadro 2.2: Análise de Centralização x Descentralização 29

Quadro 2.3: Instrumento para Seleção de Estrutura 31

Quadro 2.4: Modelos de Negócios Eletrônicos segundo Moore 49

Quadro 2.5: Níveis de Descentralização de NE segundo Vasconcellos \& Muritiba 53

Quadro 3.1: Métodos de Pesquisa 59

Quadro 3.2: Roteiros de Entrevista Utilizados em Cada Fase da Pesquisa 64

Quadro 4.1: Unidades de Negócio da Empresa X 70

Quadro 4.2: Critérios para Análise dos Graus de Descentralização 87

Quadro 4.3: Roteiro de entrevista Q3, para avaliar cada alternativa de estrutura segundo os critérios identificados 88

Quadro 4.4: Resultados individuais da avaliação das alternativas de estrutura 90

Quadro 4.5: Médias das Respostas Obtidas 91

Quadro 4.6: Notas Ponderadas para os Diferentes Graus de Centralização 92

Quadro 4.7: Diferenças das Notas Ponderadas com as Médias 94

Quadro 4.8: Critérios Ordenados Para a Alternativa 1 95

Quadro 4.9: Critérios Ordenados Para a Alternativa 2 100

Quadro 4.10: Critérios Ordenados Para a Alternativa 3 104

Quadro 5.1: Iniciativas / Aplicações de NE na Empresa X 112

Quadro 5.2: Atribuições das Áreas Envolvidas no Processo de NE 114

Quadro 5.3: Identificação das BUs pelas atribuições desempenhadas 116

Quadro 5.4: Pontuação Final dos Graus de Descentralização de NE 118

Quadro 5.5: Critérios que mais favorecem cada alternativa de estrutura para CE_ 118 Quadro 5.6: Metodologia Utilizada para Análise dos Graus de Descentralização de NE120 Quadro 7.1: Relacionamento literatura $x$ assunto em negócios eletrônicos 137 


\section{Resumo}

Nesta dissertação foram estudadas as alternativas de estrutura organizacional que podem ser adotadas para a realização de negócios eletrônicos (e-business) em empresas tradicionais, enfocando principalmente a análise sobre centralização ou descentralização desta estrutura.

$\mathrm{Na}$ pouca literatura existente especificamente sobre estrutura organizacional para negócios eletrônicos, fica claro que existe um leque de opções em termos de alternativas de estrutura, desde criar uma empresa "ponto com" totalmente separada, até descentralizar os negócios eletrônicos pelas várias unidades de negócio.

No caso estudado nesta dissertação, inicialmente foram identificadas as iniciativas de negócios eletrônicos praticadas pela empresa, sendo discutido o processo de adoção de tais ferramentas.

Depois, foi analisada a evolução da estrutura criada para negócios eletrônicos desde o seu início até o momento em que a pesquisa foi realizada. Foi identificado que as atribuições delineadas inicialmente para os negócios eletrônicos não são rígida e uniformemente desempenhadas pelas várias unidades de negócio.

Como a empresa estudada passava justamente por um período de discussão sobre descentralizar o Departamento de eCommerce, o momento foi altamente apropriado para a realização da análise sobre centralização e descentralização.

A empresa identificou três alternativas de estrutura, bem como os critérios para uma análise aprofundada. A alternativa de estrutura de eCommerce centralizado foi a mais recomendada, dentro das limitações da metodologia, devidamente apresentadas e discutidas no texto.

Mais do que a recomendação final da análise realizada, que serve apenas para a empresa pesquisada, a metodologia utilizada é uma das grandes contribuições desta dissertação. Com as devidas adaptações, ela pode ser útil em outras situações reais, auxiliando assim os gestores nesta complexa decisão sobre qual o grau de descentralização a ser adotado nos negócios eletrônicos. 


\section{Abstract}

Were studied in this dissertation the alternatives in organizational structure which could be adopted in electronic business (e-business) in traditional companies, mainly focusing this structure's centralization or decentralization analysis.

In the brief existing bibliography specifically about organizational structure for ebusiness, it is clear that exists a range of options in terms of structure choices, from creating an independent "dot com" company, to the decentralization of the e-business through the business units.

In the case studied in this dissertation, firstly were identified the e-business initiatives practiced in the company and were discussed the adoption process of these tools.

After that, the evolution of the structure created for e-business was analyzed, from the beginning to the application moment of this research. It was possible to identify that the initially delineated attributions for e-business are not rigid and are not uniformly realized by all of the business units.

As the studied company was discussing the decentralization of the eCommerce Department, the timing for the centralization or decentralization analysis was very appropriate.

The company identified three alternatives of structure, as well as the criterions for an in depth analysis. The centralized eCommerce structure alternative was the most recommended, considering the limitations of the methodology, properly presented and discussed in the text.

More than the final recommendation of the analysis made, which interests only for the studied company, the applied methodology is one of the mainly contributions of this dissertation. With the needed modifications, this methodology can be useful in other real situations, helping the managers in this complex decision about which degree of decentralization to adopt in e-business. 